ARTIGO ORIGINAL ORIGINAL ARTICLE

\title{
Análise econômica do tratamento de tromboembolismo venoso com rivaroxabana em comparação com enoxaparina seguida de varfarina sob a perspectiva do Sistema de Saúde Suplementar brasileiro
}

\author{
Economic analysis of the treatment of venous thromboembolism \\ with rivaroxaban in comparison with enoxaparin plus warfarin \\ under the perspective of the Brazilian Private Healthcare System
}

Alexandra David Piedade', Luciano Paladini', Diego Kashiura',

Teresa Lemmer ${ }^{1}$, Flávia Sauer Tobaruella², Karisa Oliveira M. Campos²

DOI: 10.21115/JBES.v9.n1.p109-21

\section{Palavras-chave:}

rivaroxabana, enoxaparina, varfarina, custos, Brasil, Saúde Suplementar

\section{Keywords:}

rivaroxaban, enoxaparin, warfarin, costs, Brazil, Private Healthcare System

\section{RESUMO}

Objetivo: $O$ tromboembolismo venoso (TEV) está associado a um grande impacto em saúde. A rivaroxabana é uma das opções recomendadas para o tratamento de TEV, segundo diretrizes internacionais (American College of Chest Physicians - ACCP 2016) (Kearon et al., 2016). É um anticoagulante oral, inibidor direto do fator Xa, que possui a vantagem de não requerer monitoramento e ajuste de dose de acordo com parâmetros laboratoriais de coagulação. O objetivo deste estudo foi comparar os custos diretos da rivaroxabana com a combinação de enoxaparina/varfarina e estimar o impacto orçamentário de sua introdução no Sistema de Saúde Suplementar brasileiro. Métodos: Realizaram-se análises de custo-minimização e impacto orçamentário comparando os tratamentos com rivaroxabana e enoxaparina/varfarina, em um horizonte temporal de um ano, sob a perspectiva do Sistema de Saúde Suplementar brasileiro. Foram considerados custos médicos diretos durante o período de internação hospitalar (medicamentos, hospitalização, acompanhamento ambulatorial e eventos adversos). A população de pacientes elegíveis foi estimada a partir de uma abordagem epidemiológica, considerando o sistema de saúde privado como um todo e para diferentes portes de operadoras de saúde. Resultados: Estimou-se que o tratamento de TEV com rivaroxabana pode gerar $\mathrm{R} \$ 1.996,99$ de economia comparado ao tratamento com enoxaparina/varfarina, principalmente devido à redução do tempo de hospitalização. O impacto orçamentário da introdução da rivaroxabana no Sistema de Saúde Suplementar apresentou economia de recursos potencial em todos os cenários analisados. A robustez do modelo foi testada por análises de sensibilidade determinísticas e a economia de recursos promovida pela rivaroxabana foi mantida em todas as variações. Conclusão: A rivaroxabana é uma alternativa para o tratamento de TEV que possui o potencial de gerar economia de recursos ao Sistema de Saúde Suplementar brasileiro.
Recebido em: 02/03/2017. Aprovado para publicação em: 20/04/2017.

1. Evidências - Kantar Health

2. Bayer Pharmaceuticals, São Paulo, SP, Brasil.

Financiamento: Este estudo teve suporte financeiro da Bayer Pharmaceuticals para a sua realização.

Conflitos de interesse: Alguns dos autores são funcionários da Bayer Pharmaceuticals, São Paulo, SP, Brasil.

Congressos: Dois pôsteres apresentados no congresso: ISPOR $18^{\text {th }}$ Annual European Congress. Milan, Italy. November, 2015

Autor correspondente: Flávia Sauer Tobaruella. Rua Domingos Jorge, 1100, prédio 501, $8^{\circ}$ andar - Socorro, São Paulo/SP -

04779-900. Telefone: +55 (11) 5694-4224. Fax: 55 (11) 5694-8053. E-mail: flavia.tobaruella@bayer.com 
formed comparing rivaroxaban versus enoxaparin/warfarin, in one-year time horizon and under the perspective of the Brazilian Private Healthcare System. Medical direct costs were considered for the hospitalization period (drugs, hospitalization, outpatient care and adverse events). Eligible patients were estimated through an epidemiological approach for the healthcare system and stratified by health management organization profile. Results: Estimated total cost of VTE treatment with rivaroxaban may cause a $\mathrm{R} \$ 1.996,99$ economy comparing to enoxaparin/warfarin, mainly due to fewer hospitalization days. The budget impact of the reimbursement of rivaroxaban in the Brazilian Private Healthcare System presented potential resource in all scenarios evaluated. Robustness of the model was tested by deterministic sensitivity analysis in which the resource saving promoted by rivaroxaban was maintained in all variations. Conclusion: Rivaroxaban is an alternative for the treatment of VTE that has potential to promote resource for the Brazilian Private Healthcare System.

\section{Introdução}

O tromboembolismo venoso (TEV) é uma doença cardiovascular que abrange a trombose venosa profunda (TVP) e sua maior complicação, a embolia pulmonar (EP). O TEV é uma grande causa mundial de morbidade e mortalidade, e sua incidência varia entre 104 e 183 casos por 100.000 pessoas/ano na população de ancestralidade europeia. A incidência reportada de TVP varia de 45 a 117 casos por 100.000 pessoas/ ano e a de EP com ou sem TVP, de 29 a 78 casos por 100.000 pessoas/ano (Heit, 2015). Os custos de tratamento são crescentes, uma vez que o manejo de TEV envolve um período de hospitalização e há risco de recorrência após o tratamento (Fernandez et al., 2015; Heit, 2015; Monreal et al., 2015).

Mais recentemente, novos agentes foram aprovados para tratamento e prevenção de recorrência de TVP e EP (Agnelli et al., 2013a; Agnelli et al., 2013b; Bauersachs et al., 2010; Büller et al., 2012; Schulman et al., 2014; Schulman et al., 2009; Schulman et al., 2013).

O aspecto principal do tratamento de TEV é a terapia anticoagulante. A anticoagulação com heparina não fracionada (HNF) e heparina de baixo peso molecular (HBPM), como a enoxaparina, em conjunto com e seguida por anticoagulação oral com antagonistas da vitamina K (AVK) foram os pilares do tratamento de TEV, tanto TVP quanto EP, ao longo dos últimos anos. Após o tratamento inicial, recomenda-se terapia anticoagulante oral para prevenção de recorrência de $T E V$, sendo uma das opções os antagonistas de vitamina $K$ (AVK), como a varfarina (Guyatt et al., 2012; Hirsh et al., 2001a; Hirsh et al., 2001b; Kearon et al., 2016; McRae \& Ginsberg, 2004).

As principais limitações da terapia com AVK são a necessidade de monitoramento constante e o ajuste de dose durante o tratamento a longo prazo.

Duas novas classes de anticoagulantes orais foram desenvolvidas com o objetivo de minimizar essas limitações: os inibidores sintéticos, seletivos e reversíveis de fator Xa (FXa) e os inibidores de trombina. A rivaroxabana é um inibidor direto seletivo do FXa cuja atividade inibe de forma direta e seletiva o fator Xa, que desempenha papel central na cascata de coagulação sanguínea. Seu efeito anticoagulante previsível permite a administração de doses fixas e a não necessidade de monitoramento laboratorial da anticoagulação para ajuste de dose (Becattini \& Agnelli, 2016).

A eficácia e a segurança da rivaroxabana no tratamento de TVP e EP foram avaliadas nos estudos clínicos EINSTEIN de fase III, que demonstraram a não inferioridade da rivaroxabana comparada ao tratamento com enoxaparina seguido de AVK, com redução de sangramento maior de forma significativa no grupo da rivaroxabana comparado ao tratamento-padrão na análise combinada do EINSTEIN TVP e EP (Prins et al., 2013). Além de apresentar eficácia semelhante ao comparador, a rivaroxabana apresentou o benefício de diminuir o tempo de hospitalização dos pacientes (Bauersachs et al., 2010; Büller et al., 2012; van Bellen et al., 2014). De acordo com as últimas recomendações das diretrizes do ACCP 2016, os NOACs (novel oral anticoagulants) são o tratamento preferencial com relação ao AVK, para TEV não associado a câncer (grau de recomendação 2B) (Kearon et al., 2016).

O estudo observacional multicêntrico, internacional e prospectivo XALIA avaliou a efetividade e a segurança da rivaroxabana, comparada com HNF, HBPM ou fondaparinux seguidos de AVK para o tratamento de TVP concomitante ou não com EP. Os desfechos primários de sangramento maior, TEV recorrente e mortalidade por todas as causas não apresentaram diferenças estatisticamente significativas entre os grupos do estudo. Porém, o tempo médio de hospitalização dos pacientes do grupo rivaroxabana foi significativamente menor do que no grupo comparador (5,0 dias versus 7,7 dias, $p<0,001$ ) (Ageno et al., 2016).

Há poucos dados sobre os custos associados ao tratamento de TEV no Brasil. Dessa forma, o objetivo deste estudo é realizar uma análise de custo-minimização da rivaroxabana em comparação com enoxaparina em combinação com varfarina (E/V) para o tratamento de TEV, bem como estimar o impacto orçamentário da sua incorporação sob a perspectiva do sistema de saúde privado brasileiro.

\section{Métodos}

\section{Racional clínico}

Dois estudos clínicos abertos, randomizados, multicêntricos, internacionais e de não inferioridade - EINSTEIN-TVP e 
EINSTEIN-EP - avaliaram se a terapia com rivaroxabana oral é não inferior à terapia com enoxaparina subcutânea seguida de AVK (varfarina ou acenocumarol) para o tratamento de TVP e EP sintomáticas, respectivamente. Esses dois estudos foram selecionados para o desenvolvimento do racional dessa análise de custo-minimização e impacto orçamentário, por serem os estudos pivotais de rivaroxabana no tratamento de TEV, avaliarem os comparadores para o tratamento de TVP e EP quanto à eficácia e segurança e serem multicêntricos, randomizados e com grande amostra de pacientes. Ambos consideraram a recorrência de TEV como desfecho primário de eficácia e a ocorrência de sangramento maior ou não maior clinicamente relevante como desfecho primário de segurança (Bauersachs et al., 2010; Büller et al., 2012).

Ambos os estudos apresentaram resultados dos desfechos primários de eficácia com intervalos de confiança (IC) de 95\% que confirmaram a não inferioridade da monoterapia com rivaroxabana (15 mg duas vezes ao dia por três semanas, seguida por 20 mg uma vez ao dia) comparada à terapia com enoxaparina (duas vezes ao dia por pelo menos cinco dias) em combinação com AVK, até relação normalizada internacional $(\mathrm{RNI}) \geq 2$ em duas medições consecutivas com pelo menos 24 horas de intervalo, seguida então de AVK sozinho por 3, 6 ou 12 meses de tratamento, a critério do médico assistente.

No estudo EINSTEIN-TVP foram incluídos 3.449 pacientes diagnosticados com TVP aguda e sintomática, sem embolia pulmonar sintomática. A recorrência de TEV foi observada em 2,1\% dos pacientes do grupo rivaroxabana e em 3,0\% no grupo enoxaparina em combinação com AVK [hazard ratio $(H R)=0,68$; IC 95\%: 0,44-1,04; $\mathrm{p}<0,001$ para não inferioridade]. O desfecho principal de segurança ocorreu em $8,1 \%$ dos pacientes em ambos os grupos ( $H R=0,97$; IC 95\%: 0,76-1,22; $p=0,77$ ) (Bauersachs et al., 2010).

No EINSTEIN-EP foram incluídos 4.832 pacientes. A rivaroxabana foi não inferior a enoxaparina/AVK quanto ao desfecho primário de eficácia, TEV recorrente sintomático, com taxas de ocorrência de 2,1\% no grupo rivaroxabana e de 1,8\% no grupo comparador ( $H R=1,12 ;$ IC 95\%: 0,75-1,68; $p=0,003$ ). Quanto ao desfecho principal de segurança, não houve diferença estatisticamente significativa entre os grupos para o primeiro episódio de sangramento maior ou não maior clinicamente relevante ( $H R=0,90 ;$ IC 95\%: 0,76-1,07; $p=0,23$ ), porém, com relação às taxas de sangramento maior, houve redução significativa no grupo rivaroxabana comparado ao tratamento-padrão ( $H R=0,49$; IC 95\%: 0,31-0,79; $p=0,003$ ) (Büller et al., 2012).

Van Bellen e Bamber conduziram análises baseadas nos dados dos estudos EINSTEIN-TVP e EINSTEIN-EP, que demonstraram redução do tempo de internação hospitalar dos pacientes tratados com rivaroxabana comparados com os tratados com enoxaparina/AVK. Observou-se que, de forma geral, 52\% dos pacientes com TVP e 90\% dos pacientes com EP são internados no evento-índice. Para pacientes com TVP, a mediana do tempo de hospitalização foi de cinco dias para o grupo rivaroxabana e de oito dias para o comparador ( $p<$ 0,0001 ). Nos pacientes com EP, as medianas foram de seis e sete dias para rivaroxabana e para o comparador, respectivamente ( $p$ <,0001) (Bauersachs et al., 2010; Büller et al., 2012; van Bellen et al., 2014).

\section{Análise de custo-minimização}

\section{Estrutura}

Segundo a Diretriz Metodológica de Avaliação Econômica brasileira, recomenda-se a realização de um estudo de custo-minimização quando há forte evidência de efetividade equivalente entre as intervenções comparadas (Brasil, 2014).

As evidências disponíveis demonstraram que não há disparidades clínicas em questões de eficácia entre os benefícios conferidos pelo uso da rivaroxabana em comparação com enoxaparina/varfarina para o tratamento de TEV (Bauersachs et al., 2010; Büller et al., 2012).

O modelo econômico proposto para a análise de custo-minimização avaliou a diferença de custos médicos diretos no ambiente hospitalar entre os tratamentos com rivaroxabana e enoxaparina/varfarina (Figura 1). Os comparadores foram selecionados de acordo com os estudos EINSTEIN, sendo considerados os custos diretos (medicamentos, consultas e procedimentos) relacionados aos tratamentos, cujas fontes estão descritas na seção "Uso de recursos e custos". Uma vez que os estudos clínicos acompanharam os pacientes durante o tempo pretendido de tratamento, ou seja, por até 12 meses, com período de 30 dias de observação após o término do tratamento, e nesse período não houve diferença estatisticamente significativa quanto à eficácia entre os grupos, considerou-se para o custo-minimização um horizonte de tempo de 12 meses. Assumiu-se assim que os custos após a terapia anticoagulante seriam semelhantes e, portanto, não foram considerados na análise.

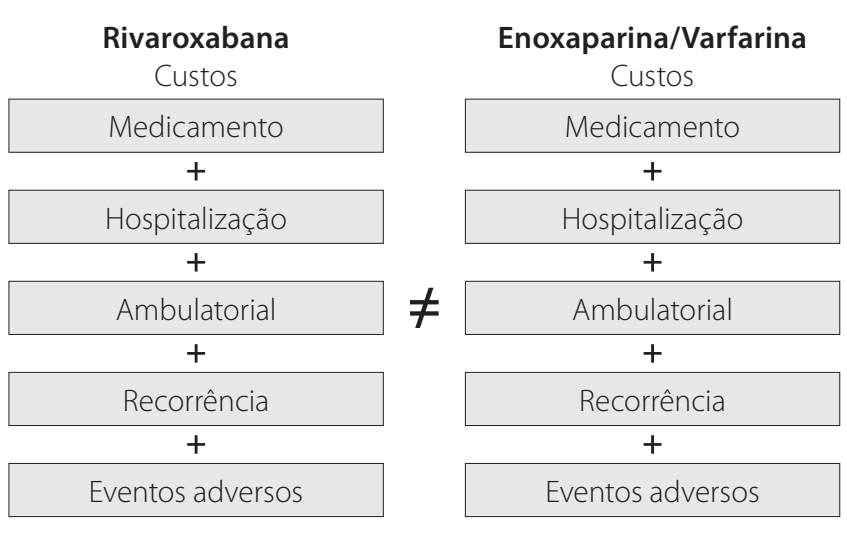

Figura 1. Estrutura do modelo de custo-minimização. 


\section{Esquema terapêutico}

Os regimes de tratamento e suas respectivas durações foram baseados naqueles observados nos estudos EINSTEIN. Para o grupo tratado com rivaroxabana, considerou-se que o regime se inicia com a posologia de $15 \mathrm{mg}$ duas vezes ao dia por três semanas, seguida de 20 mg uma vez ao dia durante o restante do período de tratamento. Para o grupo comparador, considerou-se um regime posológico de enoxaparina em combinação com AVK até RNI $\geq 2$, seguido de AVK sozinho durante o restante do período de tratamento. A duração do tratamento com enoxaparina em conjunto com varfarina foi considerada a mediana descrita nos estudos EINSTEIN de oito dias tanto para TVP quanto para EP (Bauersachs et al., 2010; Büller et al., 2012).

\section{Uso de recursos e custos}

Para a estimativa do uso de recursos, foram combinados os dados extraídos de estudos clínicos e os resultados de uma pesquisa virtual realizada com especialistas e descrita em detalhes ao final desta sessão.

As taxas de ocorrência dos desfechos clínicos foram extraídas dos estudos EINSTEIN e estão descritas na Tabela 1. As taxas de ocorrência de sangramentos intracraniano e retroperitoneal foram obtidas a partir da soma desses eventos fatais e não fatais divididos pelo número de pacientes em cada braço. Os demais valores de sangramentos e recorrência de TEV foram obtidos diretamente das taxas reportadas pelos estudos.

Os tempos de hospitalização dos pacientes dos dois grupos foram extraídos da análise dos estudos EINSTEIN-TVP e EINSTEIN-EP. Para pacientes com TVP, a mediana de hospita-

Tabela 1. Taxa de ocorrência dos desfechos clínicos considerados na análise econômica

\begin{tabular}{lcccc}
\hline & \multicolumn{4}{c}{ Taxa de ocorrência (\%) } \\
\cline { 2 - 5 } & \multicolumn{3}{c}{ TVP } & \multicolumn{3}{c}{ EP } \\
\cline { 2 - 5 } Desfecho clínico & $\mathbf{R}$ & E/V & $\mathbf{R}$ & E/V \\
\hline $\begin{array}{l}\text { Sangramento } \\
\text { intracraniano }\end{array}$ & $\begin{array}{c}\text { Não } \\
\text { reportado }\end{array}$ & $\begin{array}{c}\text { Não } \\
\text { reportado }\end{array}$ & $\begin{array}{c}0,12 \\
(3 / 2412)\end{array}$ & $\begin{array}{c}0,5 \\
(12 / 2405)\end{array}$ \\
\hline $\begin{array}{l}\text { Sangramento } \\
\text { retroperitoneal }\end{array}$ & 0 & 0 & $\begin{array}{c}0,04 \\
(1 / 2412)\end{array}$ & $\begin{array}{c}0,33 \\
(8 / 2402)\end{array}$ \\
\hline $\begin{array}{l}\text { Sangramento } \\
\text { maior }\end{array}$ & 0,8 & 1,2 & 1,1 & 2,2 \\
\hline $\begin{array}{l}\text { Sangramento } \\
\text { não maior } \\
\text { clinicamente } \\
\text { relevante }\end{array}$ & 7,3 & 7,0 & 9,5 & 9,8 \\
\hline $\begin{array}{l}\text { TEV recorrente } \\
\text { Síndrome pós- }\end{array}$ & 2,1 & 3,0 & 2,1 & 1,8 \\
\hline \begin{tabular}{l} 
trombótica \\
\hline
\end{tabular}
\end{tabular}

TVP: trombose venosa profunda; EP: embolia pulmonar; R: rivaroxabana; E/V: enoxaparina mais varfarina; TEV: tromboembolismo venoso. lização foi de 5,0 dias [amplitude interquartil (Al): 3,0-9,0] para o grupo rivaroxabana e 8,0 dias (Al 4,0-10,0) para o comparador. No tratamento de EP, as medianas foram de 6,0 dias (AI 4,0-9,0) e 7,0 dias (Al 5,0-10,0), respectivamente (van Bellen et al., 2014).

A taxa de síndrome pós-trombótica (SPT) foi extraída de uma análise post-hoc dos estudos EINSTEIN, que mostrou incidência cumulativa em 60 meses de 29\% no grupo rivaroxabana e de $40 \%$ no grupo enoxaparina/AVK, porém não houve diferença estatisticamente significativa entre os dois grupos. Para 12 meses, as incidências cumulativas foram estimadas, a partir da curva de Kaplan-Meier, em 17,9\% e 19,6\%, para rivaroxabana e enoxaparina/AVK, respectivamente (Cheung et al., 2016). Um estudo observacional internacional que acompanhou o histórico clínico de pacientes com TVP encontrou uma taxa semelhante de 17,3\%, depois de 12 meses de seguimento após o evento-índice, validando, assim, o dado incluído (Prandoni et al., 1996). Esse mesmo estudo mostrou que, desses eventos, 30,2\% são considerados graves, valor esse assumido no modelo (Prandoni et al., 1996).

De acordo com os dados apresentados pelo estudo EINSTEIN-EP, 24,75\% dos pacientes com diagnóstico primário de EP também apresentavam TVP e teriam potencial de desenvolver SPT. Assim, os valores de 17,95\% e 19,60\% foram ponderados pela taxa de $24,75 \%$, obtendo-se as ocorrências de 4,4\% e 4,8\% de STP na população com diagnóstico primário de EP.

Para a estimativa do uso de recursos referentes ao tratamento de TEV, TVP e EP e ao manejo de eventos adversos relacionados às terapias com rivaroxabana e enoxaparina/ varfarina, foi realizada uma pesquisa com sete profissionais, entre os quais se encontravam especialistas em auditoria de requerimento de intervenções em saúde, oncologia clínica, cirurgia vascular, terapia intensiva, cirurgia geral e geriatria. A pesquisa foi composta por uma série de perguntas, com o objetivo de obter dados sobre a prática de tratamento e acompanhamento dos pacientes. Com base nas respostas, foram calculadas as médias das frequências de uso dos recursos relacionados na pesquisa para hospitalização e acompanhamento ambulatorial, descritos na Tabela 2.

Para os pacientes que apresentaram sangramento intracraniano e sangramento retroperitoneal, o uso de recursos foi estimado pela pesquisa com os especialistas via e-mail (Tabela 3). Para os demais pacientes com sangramento maior, o consumo de recursos foi definido como uma visita ao departamento de emergência mais transfusão de 1,5 unidade internacional (UI) de concentrado de hemácias por paciente. Assumimos que os eventos de sangramento não maior clinicamente relevante são manejados apenas com uma visita médica adicional para cada evento e um teste adicional de RNI. Para testar o impacto das incertezas dos dados utilizados, esses parâmetros foram variados na análise de sensibili- 
Tabela 2. Resultados da pesquisa com especialistas realizada para estimativa do uso de recursos utilizados na hospitalização e acompanhamento ambulatorial

\begin{tabular}{|c|c|c|c|c|c|c|c|c|c|c|c|c|c|c|c|c|c|c|c|c|}
\hline & \multicolumn{4}{|c|}{ Hospitalização } & \multicolumn{4}{|c|}{$\begin{array}{l}\text { Acompanhamento } \\
\text { Ambulatorial } \\
\text { até o } 10^{\circ} \mathrm{dia}\end{array}$} & \multicolumn{12}{|c|}{ Acompanhamento Ambulatorial a partir do $10^{\circ}$ dia } \\
\hline & & & \multirow{2}{*}{\multicolumn{2}{|c|}{ EP }} & \multirow{2}{*}{\multicolumn{2}{|c|}{ TVP }} & \multirow{2}{*}{\multicolumn{2}{|c|}{ EP }} & \multicolumn{6}{|c|}{ TVP } & \multicolumn{6}{|l|}{ EP } \\
\hline & \multicolumn{2}{|l|}{ TVP } & & & & & & & \multicolumn{2}{|c|}{3 meses } & \multicolumn{2}{|c|}{6 meses } & \multicolumn{2}{|c|}{12 meses } & \multicolumn{2}{|c|}{3 meses } & \multicolumn{2}{|c|}{6 meses } & \multicolumn{2}{|c|}{12 meses } \\
\hline & $\mathbf{R}$ & $E / V$ & $\mathbf{R}$ & $\mathrm{E} / \mathrm{V}$ & $\mathbf{R}$ & $E / V$ & $\mathbf{R}$ & $\mathrm{E} / \mathrm{V}$ & $\mathbf{R}$ & $\mathrm{E} / \mathrm{V}$ & $\mathbf{R}$ & $\mathrm{E} / \mathrm{V}$ & $\mathbf{R}$ & $\mathrm{E} / \mathrm{V}$ & $\mathbf{R}$ & $\mathrm{E} / \mathrm{V}$ & $\mathbf{R}$ & $\mathrm{E} / \mathrm{V}$ & $\mathbf{R}$ & $E / V$ \\
\hline $\begin{array}{l}\text { US duplex de } \\
\text { membros inferiores }\end{array}$ & 1 & 1 & 1 & 1 & 1 & 1 & 1 & 1 & 0 & 0 & 1 & 1 & 1 & 1 & 0 & 0 & 1 & 1 & 1 & 1 \\
\hline Flebografia & 0,1 & 0,1 & 0,3 & 0,3 & 0,1 & 0,1 & 0,1 & 0,1 & - & - & - & - & - & - & - & - & - & - & - & - \\
\hline TTPa & 0 & 4 & 0 & 7 & 0 & 3 & 0 & 3 & - & - & - & - & - & - & - & - & - & - & - & - \\
\hline $\mathrm{RNI}$ & 0 & 4 & 0 & 7 & 0 & 3 & 0 & 3 & 0 & 4 & 0 & 7 & 0 & 13 & 0 & 4 & 0 & 7 & 0 & 13 \\
\hline Hemograma & 0 & 3 & 2 & 7 & 0 & 1 & 1 & 1 & 0 & 4 & 0 & 7 & 0 & 12 & 0 & 4 & 0 & 7 & 0 & 12 \\
\hline Dímero D & 1 & 1 & 1 & 1 & 0,2 & 0,2 & 1 & 1 & - & - & - & - & - & - & - & - & - & - & - & - \\
\hline Rx de tórax & 1 & 1 & 1 & 1 & 1 & 1 & 1 & 1 & - & - & - & - & - & - & - & - & 1 & 1 & 1 & 1 \\
\hline US de abdome & 0,3 & 0,3 & 1 & 1 & 1 & 1 & - & - & - & - & - & - & - & - & - & - & - & - & - & - \\
\hline ECG & - & - & 1 & 1 & - & - & 1 & 1 & - & - & - & - & - & - & - & - & - & - & - & - \\
\hline Ecocardiograma & - & - & 0,6 & 0,6 & - & - & 0,6 & 0,6 & - & - & - & - & - & - & 0,5 & 0,5 & 0,7 & 0,7 & 1,4 & 1,4 \\
\hline $\mathrm{CT}$ de tórax multislice & - & - & 1 & 1 & - & - & 1 & 1 & - & - & - & - & - & - & 1 & 1 & 1 & 1 & 1 & 1 \\
\hline $\begin{array}{l}\text { Cintilografia } \\
\text { ventilação-perfusão }\end{array}$ & - & - & 0,2 & 0,2 & - & - & 0,2 & 0,2 & - & - & - & - & - & - & - & - & - & - & - & - \\
\hline Arteriografia pulmonar & - & - & 0,1 & 0,1 & - & - & 0,05 & 0,05 & - & - & - & - & - & - & - & - & - & - & - & - \\
\hline UTI (diária/paciente) & - & - & 0,8 & 0,8 & - & - & - & - & - & - & - & - & - & - & - & - & - & - & - & - \\
\hline Gasometria arterial & - & - & 4 & 4 & - & - & - & - & - & - & - & - & - & - & - & - & - & - & - & - \\
\hline Visitas médicas & - & - & - & - & 1 & 1 & 1 & 1 & 2 & 4 & 3 & 7 & 6 & 13 & 3 & 4 & 5 & 7 & 9 & 13 \\
\hline
\end{tabular}

TVP: trombose venosa profunda; EP: embolia pulmonar; R: rivaroxabana; E/V:enoxaparina mais varfarina; TEV: tromboembolismo venoso; TTPa: tempo de tromboplastina parcial ativada; RNI: relação normalizada internacional; Rx: raio X; CT: tomografia computadorizada; UTI: unidade de terapia intensiva.

Tabela 3. Resultados da pesquisa com especialistas realizada para estimativa do uso de recursos utilizados no manejo de sangramento maior

\begin{tabular}{|c|c|c|c|}
\hline & \multirow{2}{*}{$\begin{array}{l}\text { Proporção } \\
\text { que recebe } \\
\text { tratamento } \\
\text { cirúrgico (\%) }\end{array}$} & \multicolumn{2}{|c|}{$\begin{array}{l}\text { Número de diárias } \\
\text { de internação }\end{array}$} \\
\hline & & $\begin{array}{l}\text { Pacientes } \\
\text { operados }\end{array}$ & $\begin{array}{c}\text { Pacientes não } \\
\text { operados }\end{array}$ \\
\hline $\begin{array}{l}\text { Sangramento } \\
\text { intracraniano }\end{array}$ & 3 & 10 & 5 \\
\hline $\begin{array}{l}\text { Sangramento } \\
\text { retroperitoneal }\end{array}$ & 1 & 7 & 3 \\
\hline RM de crânio & - & 3 & 2 \\
\hline RM de abdome & - & 3 & 2 \\
\hline $\begin{array}{l}\text { Concentrado } \\
\text { de hemácias }\end{array}$ & \multicolumn{3}{|c|}{ 1,5 UI/paciente operado ou não } \\
\hline
\end{tabular}

RM: ressonância magnética.

dade determinística. Os recursos utilizados para o manejo de TEV recorrente foram os mesmos do evento-índice, assumindo que os pacientes reiniciam a terapia de anticoagulação.

O consumo de recursos para SPT descritos na Tabela 4 foram estimados com base em dados coletados em estudo prospectivo observacional conduzido no Brasil (Ramacciotti et al., 2006). Os custos com cirurgia para SPT não serão considerados dado o período de incidência da complicação em relação ao tempo de permanência do paciente no modelo (Ramacciotti et al., 2006).

Para esta análise foram considerados apenas custos médicos diretos relacionados ao tratamento da doença ou de desfechos clínicos. Gastos hospitalares ou ambulatoriais foram incluídos, não cobrindo medicamentos em uso domiciliar. Foi realizado microcusteio para os recursos utilizados para hospitalização e acompanhamento ambulatorial dos pacientes com TEV.

Os dados dos custos dos medicamentos foram obtidos da lista da Câmara de Regulação do Mercado de Medicamentos (CMED) atualizada em dezembro de 2016, considerando Preço Fábrica 18\%. Os custos unitários dos medicamentos foram de $\mathrm{R} \$$ 6,75 por comprimido de $15 \mathrm{mg}$ de rivaroxabana. Para enoxaparina e varfarina, foi calculada a média dos preços das diferentes apresentações disponíveis, $\mathrm{R} \$$ 0,47 por comprimido de $5 \mathrm{mg}$ de varfarina e $\mathrm{R} \$ 79,96$ por seringa preenchida de 80 mg de enoxaparina (CMED, 2016). 
Tabela 4. Uso de recursos no manejo de síndrome pós-trombótica

\begin{tabular}{lcc}
\hline & \multicolumn{2}{c}{ Taxa de Uso (\%) } \\
\cline { 2 - 3 } Recurso & $\begin{array}{c}\text { Casos leves ou } \\
\text { moderados }\end{array}$ & Casos graves \\
\hline US duplex & 58,80 & 35,6 \\
\hline Venografia & 5,9 & 15,1 \\
\hline $\begin{array}{l}\text { Hospitalização } \\
\text { (média de 14,5 dias) }\end{array}$ & - & 20,5 \\
\hline $\begin{array}{l}\text { Consultas médicas } \\
\text { (número de } \\
\text { consultas) }\end{array}$ & 4,9 & 6,1 \\
\hline
\end{tabular}

O custo de procedimentos foi obtido da tabela da Classificação Brasileira Hierarquizada de Procedimentos Médicos (CBHPM) 5a edição (CBHPM, 2012). A versão atualizada da CBHPM não foi utilizada, uma vez que a de 2012 ainda é a referência utilizada para base de reembolso pela maioria das operadoras.

O custo total dos tratamentos com rivaroxabana e enoxaparina/varfarina foi obtido por meio da soma dos custos dos medicamentos, hospitalização, acompanhamento ambulatorial, recorrência de TEV e manejo dos eventos adversos em cada grupo.

\section{Análise de sensibilidade}

Para verificar o impacto da incerteza dos parâmetros utilizados no modelo de custo-minimização nos resultados apresentados, realizou-se uma análise de sensibilidade determinística univariada e bivariada.

Foram univariados os parâmetros: aumento no custo de cirurgias em 100\%; custo da rivaroxabana em $\pm 7 \%$ devido à variação do preço do medicamento em diferentes contextos; custo da enoxaparina em $\pm 10 \%$ devido à variação do preço das diferentes opções do mercado; dias de hospitalização de acordo com uma taxa-padrão de $\pm 20 \%$.

Os custos de hospitalização e acompanhamento ambulatorial foram bivariados, pois abrangem ambos os custos, de TVP e EP, para obter o resultado de TEV. A variação foi feita em $\pm 20 \%$, de acordo com uma taxa-padrão.

\section{Análise de impacto orçamentário}

\section{Modelo}

Uma análise de impacto orçamentário (AIO) consiste na avaliação das consequências financeiras relacionadas à adoção de uma nova tecnologia em saúde em determinado cenário com recursos finitos (Brasil, 2014).

A presente AIO foi realizada sob a perspectiva do Sistema de Saúde Suplementar brasileiro, em um horizonte de tempo de um ano, para mensurar o impacto financeiro da introdução da rivaroxabana para o tratamento de TEV no ambiente hospitalar. Os custos anuais de tratamento com rivaroxabana e enoxaparina/varfarina foram extraídos da análise de custo-minimização descrita no presente estudo.

Foram considerados apenas custos diretos de cobertura por parte das operadoras de saúde. A AlO contemplou a perspectiva do Sistema de Saúde Suplementar como um todo. Uma vez que no sistema suplementar não há um pagador único, simulou-se a AlO para outras três perspectivas, de acordo com os tamanhos das operadoras de saúde: 1) operadoras de saúde de pequeno porte (< 20.000 vidas); 2 ) operadoras de saúde de médio porte (20.000 a 100.000 vidas); 3) operadoras de saúde de grande porte (> 100.000 vidas).

\section{População elegível}

A estimativa da população elegível ao tratamento de TEV no Sistema de Saúde Suplementar brasileiro foi realizada por meio do método epidemiológico. Esse método é recomendado pela Diretriz Metodológica de Análises de Impacto Orçamentário brasileira e foi selecionado por estimar de forma abrangente a população de interesse (Brasil, 2014).

De acordo com um estudo observacional retrospectivo que analisou uma coorte de 2.218 pacientes nos Estados Unidos durante um período de 25 anos, adotaram-se as taxas de incidência de TVP de 0,48 e de EP de 0,69 a cada 1.000 indivíduos (Silverstein et al., 1998). Uma revisão sistemática da literatura avaliou publicações internacionais sobre a incidência anual de TEV até maio de 2014, abrangendo países da Europa Ocidental, América do Norte, Austrália e Argentina. Os estudos reportaram incidências anuais de TEV variando entre 0,75 e 2,69 a cada 1.000 indivíduos da população. A maioria dos estudos reportou incidências na faixa de 1,07 a 1,83 casos a cada 1.000 indivíduos, o que está em linha com o valor assumido no presente estudo (Raskob et al., 2014).

Segundo a Agência Nacional de Saúde Suplementar (ANS), em 2016, o número total de beneficiários do Sistema de Saúde Suplementar era de 48.301 .677 indivíduos. Para a estimativa desse número e do número de beneficiários por porte de operadora no ano de 2017, utilizou-se a média da taxa de crescimento $(-1,8 \%)$ dos anos de 2015 e 2016 (Brasil, 2016).

\section{Market share}

Para cada perspectiva, foram determinados três cenários. No cenário 1, considerou-se a introdução da rivaroxabana e sua distribuição para todos os pacientes elegíveis (market share 100\%). No cenário 2, considerou-se um market share de 50\% para rivaroxabana e $50 \%$ para enoxaparina mais varfarina. No cenário 3, estabeleceu-se a divisão de 70\% do mercado para rivaroxabana e de $30 \%$ para enoxaparina mais varfarina.

\section{Custos e uso de recursos}

Os dados de esquema terapêutico, custos e usos de recursos foram os mesmos utilizados para a análise de custo-minimização e descritos na seção correspondente deste artigo. 


\section{Análise de sensibilidade}

Análises de sensibilidade univariada e bivariada foram realizadas para todos os cenários de market share e portes de operadoras de saúde, a fim de verificar a influência dos parâmetros do modelo nos resultados de impacto orçamentário. Os parâmetros variados de forma univariada foram a taxa de crescimento do número de beneficiários, as incidências de TVP e EP e os custos das drogas, e os de forma bivariada foram os dias de hospitalização do tratamento com as drogas. A variação em dias de hospitalização foi também realizada para o impacto orçamentário, por ter sido o parâmetro que gerou maior impacto na análise de custo-minimização.

As variações de custos e dias de hospitalização foram iguais às utilizadas na análise de sensibilidade do estudo de custo-minimização ( $\pm 20 \%)$. As médias de incidência de TVP e EP foram variadas de acordo com os seus respectivos ICs: mínimo de 0,45 e máximo de 0,51/1.000 indivíduos para TVP e mínimo de 0,65 e máximo de 0,73/1.000 indivíduos para EP (Silverstein et al., 1998). A taxa de crescimento do número de beneficiários do Sistema Suplementar de Saúde brasileiro foi variada para o mínimo de -2,7\% e máximo de -0,7\%, por terem sido os valores extremos apresentados entre os anos de 2015 e 2016 (Brasil, 2016).

\section{Resultados}

\section{Análise de custo-minimização}

\section{Cenário-base}

De acordo com as condições estabelecidas, o tratamento de TEV com rivaroxabana apresentou potencial para economia de recursos comparado ao tratamento com enoxaparina mais varfarina.

Para TVP, o tratamento com rivaroxabana tem os custos estimados para um ano em $\mathrm{R} \$ 2.910,68$, enquanto o comparador totaliza $\mathrm{R} \$ 4.981,88$. Para $E P$, a estimativa para rivaroxabana é de $R \$ 7.958,43$ e para E/V é de $R \$ 9.904,38$. Nota-se que os custos de hospitalização são os principais responsáveis pelo menor custo total de tratamento da rivaroxabana (Tabela 5).

Tabela 5. Tempo de hospitalização e custos dos tratamentos com rivaroxabana e enoxaparina/varfarina para TVP e EP

\begin{tabular}{|c|c|c|c|c|c|c|}
\hline \multirow{2}{*}{ TVP } & \multicolumn{3}{|c|}{ Rivaroxabana } & \multicolumn{3}{|c|}{ Enoxaparina/Varfarina } \\
\hline & \multicolumn{3}{|c|}{ Dias [mediana (AI)] } & \multicolumn{3}{|c|}{ Dias [mediana (Al)] } \\
\hline Tempo de hospitalização & \multicolumn{3}{|c|}{$5(3-9)$} & \multicolumn{3}{|c|}{$8(4-10)$} \\
\hline Categoria & Custo & $\begin{array}{c}\text { Taxa de } \\
\text { Ocorrência }\end{array}$ & Custo Total & Custo & $\begin{array}{c}\text { Taxa de } \\
\text { Ocorrência }\end{array}$ & Custo Total \\
\hline Hospitalização & $\mathrm{R} \$ 4.132,71$ & $52,00 \%$ & $\mathrm{R} \$ 2.149,01$ & $\mathrm{R} \$ 7.528,12$ & $52,00 \%$ & $\mathrm{R} \$ 3.914,62$ \\
\hline Ambulatorial & $\mathrm{R} \$ 1.122,97$ & $48,00 \%$ & $R \$ 539,03$ & $R \$ 1.554,45$ & $48,00 \%$ & $\mathrm{R} \$ 746,14$ \\
\hline Recorrência & $\mathrm{R} \$ 2.688,04$ & $2,10 \%$ & $R \$ 56,45$ & $\mathrm{R} \$ 4.660,76$ & $3,00 \%$ & $\mathrm{R} \$ 139,82$ \\
\hline Sangramento maior & $\mathrm{R} \$ 135,58$ & $0,80 \%$ & $\mathrm{R} \$ 1,08$ & $\mathrm{R} \$ 135,58$ & $1,20 \%$ & $R \$ 1,63$ \\
\hline Sangramento menor & $R \$ 62,84$ & $7,30 \%$ & $R \$ 4,59$ & $R \$ 62,84$ & $7,00 \%$ & $R \$ 4,40$ \\
\hline SPT & $R \$ 894,28$ & $17,95 \%$ & $R \$ 160,52$ & $R \$ 894,28$ & $19,60 \%$ & $R \$ 175,28$ \\
\hline \multirow[t]{2}{*}{ Custo Total } & & & $\mathrm{R} \$ 2.910,68$ & & & $\mathrm{R} \$ 4.981,88$ \\
\hline & \multicolumn{3}{|c|}{ Rivaroxabana } & \multicolumn{3}{|c|}{ Enoxaparina/Varfarina } \\
\hline EP & \multicolumn{3}{|c|}{ Dias (mediana [AI]) } & \multicolumn{3}{|c|}{ Dias (mediana [AI]) } \\
\hline Tempo de hospitalização & \multicolumn{3}{|c|}{$6(4-9)$} & \multicolumn{3}{|c|}{$7(5-10)$} \\
\hline Categoria & Custo & $\begin{array}{c}\text { Taxa de } \\
\text { ocorrência }\end{array}$ & Custo total & Custo & $\begin{array}{c}\text { Taxa de } \\
\text { ocorrência }\end{array}$ & Custo total \\
\hline Hospitalização & $\mathrm{R} \$ 8.270,92$ & $90,00 \%$ & $\mathrm{R} \$ 7.443,83$ & $\mathrm{R} \$ 10.344,78$ & $90,00 \%$ & $R \$ 9.310,30$ \\
\hline Ambulatorial & $R \$ 2.975,46$ & $10,00 \%$ & $R \$ 297,55$ & $R \$ 3.307,83$ & $10,00 \%$ & $\mathrm{R} \$ 330,78$ \\
\hline Recorrência & $\mathrm{R} \$ 7.741,38$ & $2,10 \%$ & $R \$ 162,57$ & $R \$ 9.641,09$ & $1,80 \%$ & $\mathrm{R} \$ 173,54$ \\
\hline Sangramento maior & $R \$ 135,58$ & $1,10 \%$ & $\mathrm{R} \$ 1,49$ & $\mathrm{R} \$ 135,58$ & $2,20 \%$ & $\mathrm{R} \$ 2,98$ \\
\hline Sangramento menor & $R \$ 62,84$ & $9,50 \%$ & $\mathrm{R} \$ 5,97$ & $R \$ 62,84$ & $9,80 \%$ & $R \$ 6,16$ \\
\hline Sangramento intracraniano & $\mathrm{R} \$ 5.007,18$ & $0,12 \%$ & $R \$ 6,01$ & $\mathrm{R} \$ 5.007,18$ & $0,50 \%$ & $R \$ 24,98$ \\
\hline Sangramento retroperitonial & $R \$ 3.678,78$ & $0,04 \%$ & $\mathrm{R} \$ 1,53$ & $R \$ 3.678,78$ & $0,33 \%$ & $R \$ 12,25$ \\
\hline SPT & $\mathrm{R} \$ 894,28$ & $4,44 \%$ & $\mathrm{R} \$ 39,73$ & $\mathrm{R} \$ 894,28$ & $4,85 \%$ & $R \$ 43,38$ \\
\hline Custo total & & & $\mathrm{R} \$ 7.958,67$ & & & $\mathrm{R} \$ 9.904,38$ \\
\hline
\end{tabular}

TVP: trombose venosa profunda; EP: embolia pulmonar; SPT: síndrome pós-trombótica, Al: amplitude interquartil. 
Para estimar o custo total de TEV com os dois comparadores, dadas as taxas de incidência anual de TVP e EP, considerou-se a ocorrência de 41,02\% dos casos sendo de TVP e de $58,97 \%$ de EP (Silverstein et al., 1998). A rivaroxabana apresentou economia de recursos de $\mathrm{R} \$ 1.996,99$ (Tabela 6). O uso de rivaroxabana propicia redução de $25 \%$ dos custos no tratamento de TEV, 42\% para TVP e 20\% para EP (Figura 2).

\section{Análise de sensibilidade}

Após a variação dos parâmetros dias de hospitalização e custos, conforme descrito na Tabela 7, todos os resultados continuaram apresentando economia de recursos para a rivaroxabana.
Os parâmetros mais sensíveis da análise foram os tempos de hospitalização de ambos os comparadores (Figura 3).

\section{Análise de impacto orçamentário}

\section{Sistema de Saúde Suplementar}

Relacionando-se os dados da ANS e os dados epidemiológicos de TEV, obteve-se um total de 32.728 indivíduos para o tratamento de EP e 22.767 para o tratamento de TVP, totalizando 55.495 indivíduos para o tratamento de TEV em todo o Sistema de Saúde Suplementar em 2017 (Figura 4A).

A AlO demonstrou que a introdução de rivaroxabana no Sistema de Saúde Suplementar completo gera economia de

Tabela 6. Resultados de custo-minimização da rivaroxabana comparada à enoxaparina/varfarina para o tratamento de TEV

\begin{tabular}{|c|c|c|c|c|}
\hline Comparador & Indicação & Custo & Taxa de ocorrência & Custo de TEV \\
\hline \multirow[t]{2}{*}{ Rivaroxabana } & TVP & $\mathrm{R} \$ 2.910,68$ & $41,02 \%$ & $\mathrm{R} \$ 1.193,96$ \\
\hline & EP & $\mathrm{R} \$ 7.958,67$ & $58,97 \%$ & $R \$ 4.693,23$ \\
\hline Total & & & & $\mathrm{R} \$ 5.887,19$ \\
\hline \multirow[t]{2}{*}{ Enoxaparina/Varfarina } & TVP & $\mathrm{R} \$ 4.981,88$ & $41,02 \%$ & $R \$ 2.043,57$ \\
\hline & $E P$ & $R \$ 9.904,38$ & $58,97 \%$ & $R \$ 5.840,62$ \\
\hline Total & & & & $\mathrm{R} \$ 7.884,18$ \\
\hline Diferença de custo & & & & $-R \$ 1.996,99$ \\
\hline
\end{tabular}

TEV: tromboembolismo venoso; TVP: trombose venosa profunda; EP: embolia pulmonar.

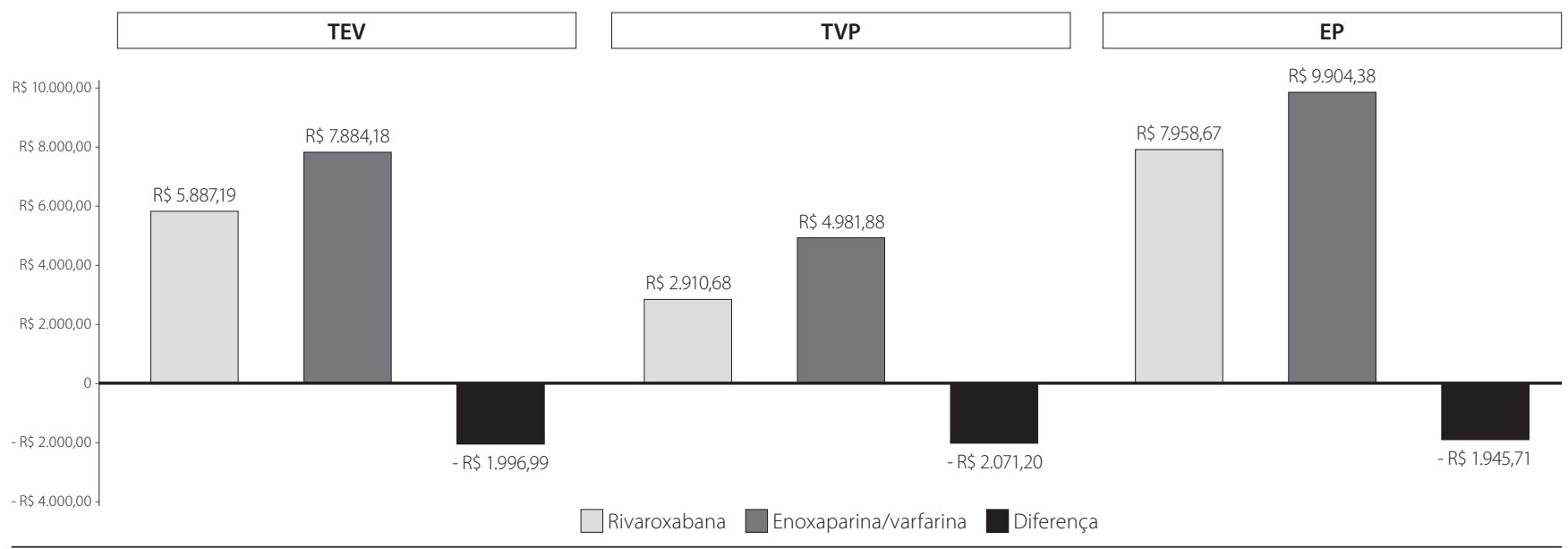

Figura 2. Custos totais e diferenças de custo no tratamento de TEV.

Tabela 7. Resultados da análise de sensibilidade do tratamento de TEV - custo-minimização

\begin{tabular}{lcccc}
\hline & Variação & Mínimo & Custo-base & Máximo \\
\hline Tempo de hospitalização (enoxaparina/varfarina) & $\pm 20 \%$ & $-R \$ 2.572,69$ & $-R \$ 1.996,99$ & $-R \$ 1.421,30$ \\
\hline Tempo de hospitalização (rivaroxabana) & $\pm 20 \%$ & $-R \$ 2.530,16$ & $-R \$ 1.996,99$ & $-R \$ 1.463,83$ \\
\hline Custos de hospitalização e ambulatoriais & $\pm 20 \%$ & $-R \$ 2.168,57$ & $-R \$ 1.996,99$ & $-R \$ 1.825,42$ \\
\hline Custo (enoxaparina) & $\pm 10 \%$ & $-R \$ 2.073,50$ & $-R \$ 1.996,99$ & $-R \$ 1.899,25$ \\
\hline Custo (rivaroxabana) & $\pm 7 \%$ & $-R \$ 2.007,15$ & $-R \$ 1.996,99$ & $-R \$ 1.999,86$ \\
\hline Aumento no custo de cirurgias & $100 \%$ & $-R \$ 1.997,08$ & $-R \$ 1.996,99$ & $-R \$ 1.996,99$ \\
\hline
\end{tabular}

TEV: tromboembolismo venoso. 
Dias de hospitalização comparador ( $\pm 20 \%)$

Dias de hospitalização rivaroxabana $( \pm 20 \%)$

Custos hospitalização e ambulatório ( $\pm 20 \%)$

Custo enoxaparina $( \pm 10 \%)$

Custo Xarelto ${ }^{\oplus}( \pm 7 \%)$

Aumento no custo de cirurgias (100\%)

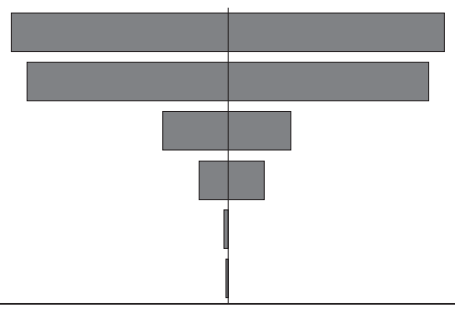

$-R \$ 2.350,00$

Figura 3. Diagrama de tornado da análise de sensibilidade - custo-minimização.

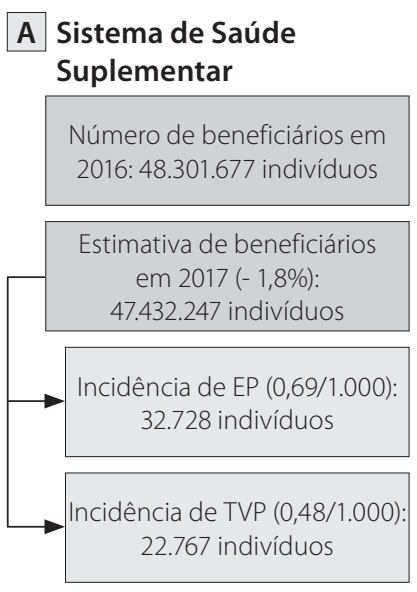

\section{B Operadora de} Pequeno Porte

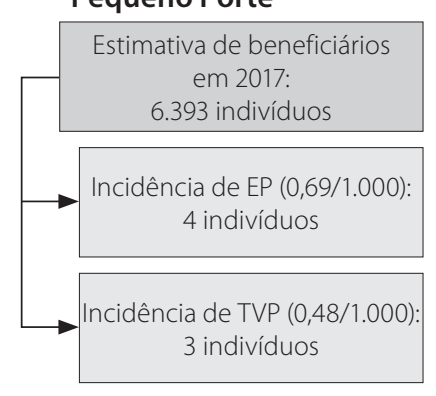

\section{Operadora de Médio Porte}

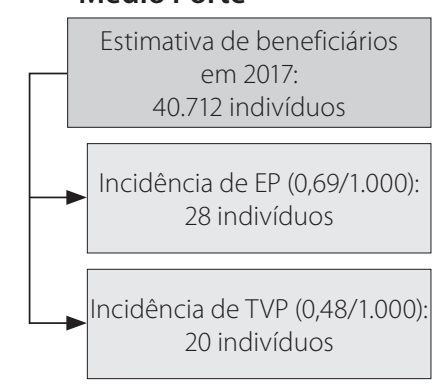

D Operadora de Grande Porte

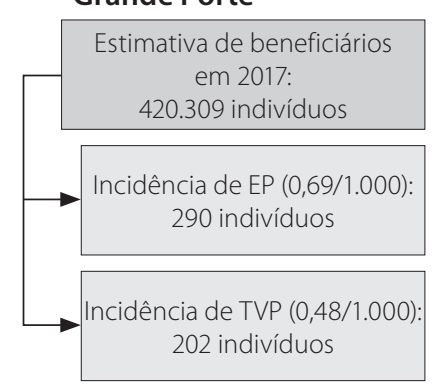

Figura 4. População elegível.

recursos nos três cenários propostos para o tratamento de TVP, EP e TEV. Com a transição completa para rivaroxabana no tratamento de TEV, há economia de R\$ 110.834.327,72. Com a transição de $50 \%$ do mercado, essa economia cai para $\mathrm{R} \$ 55.417 .163,86$. Finalmente, com market share de $70 \%$ para rivaroxabana, a economia é de $\mathrm{R} \$ 77.584 .029,40$ (Figura 5A).

\section{Operadora de saúde de pequeno porte}

Foi estimada uma média de 6.393 beneficiários cobertos por operadora de saúde de pequeno porte no ano de 2017 (Tabela 8). Desses, quatro indivíduos seriam elegíveis para o tratamento de EP e três para o tratamento de TVP (Figura 4B).

Nessas operadoras, a economia de recursos totalizou $\mathrm{R} \$ 13.996,46$ com a transição total para rivaroxabana para o tratamento de TEV, R\$ 6.998,23 para transição de 50\% e $\mathrm{R} \$$ 9.797,52 para a transição de 70\% do mercado (Figura 5B).

\section{Operadora de saúde de médio porte}

Para as operadoras de médio porte, estimou-se uma média de 40.712 beneficiários em 2017 (Tabela 8). Dos quais, 28 e 20 seriam elegíveis para o tratamento de EP e TVP, respectivamente (Figura 4C).

A introdução de rivaroxabana para o tratamento de TEV nessas operadoras, na totalidade do mercado, gerou economia de $\mathrm{R} \$$ 95.903,98; na estimativa de 50\% de uso, gerou economia de $\mathrm{R} \$$ 47.951,99; e na estimativa de 70\%, a economia foi de $R \$ 67.132,79$ (Figura 5C).

\section{Operadora de saúde de grande porte}

Para 2017, estimaram-se 420.309 beneficiários (Tabela 8), 290 elegíveis para o tratamento de EP e 202 para TVP nas operadoras de saúde de grande porte (Figura 4D).

A introdução de rivaroxabana para o tratamento de TEV pelas operadoras de saúde de grande porte apresentou as seguintes economias de recursos, de acordo com os market share de 100\%, 50\% e 70\%: R\$ 982.639,37, R\$ 491.319,68 e R\$ $687.847,56$ (Figura 5D).

\section{Impacto orçamentário por beneficiário}

Estimou-se também o impacto orçamentário por beneficiário nos três cenários de diferentes market share. No cenário 1, a introdução de rivaroxabana está relacionada à economia de $R \$$ 2,34/beneficiário para o tratamento de TEV, R\$ 0,99 para TVP e R\$1,34 para EP. No cenário 2, a sua introdução em 50\% no mercado gerou economia de $R \$ 1$ 1,17/beneficiário para TEV, R\$ 0,50 para TVP e R\$ 0,67 para EP. No cenário 3, a economia relacionada ao tratamento de TEV, TVP e EP foi de $\mathrm{R} \$ 1,64, \mathrm{R} \$ 0,70$ e $\mathrm{R} \$$ 0,94/beneficiário, respectivamente.

\section{Análise de sensibilidade}

A variação de todos os parâmetros em todas as perspectivas e cenários apresentou resultados com economia de recursos para a rivaroxabana. Uma mesma amplitude de variação foi causada para os três cenários, por isso estes estão descritos 
A

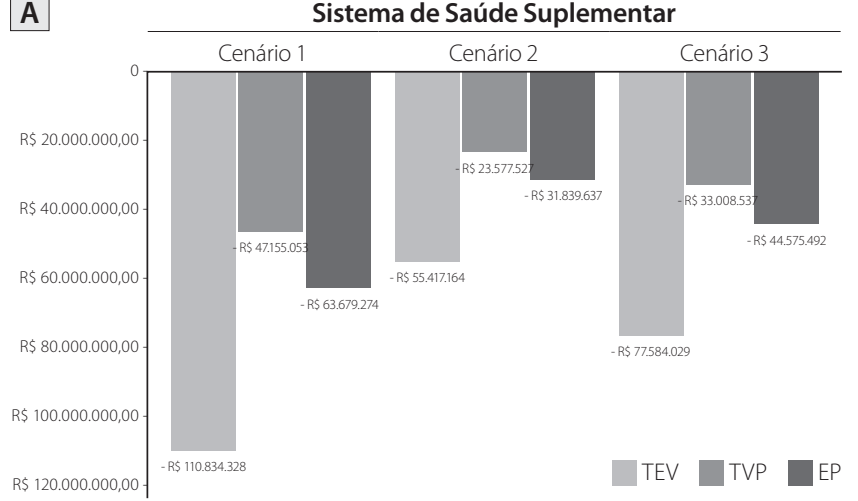

C

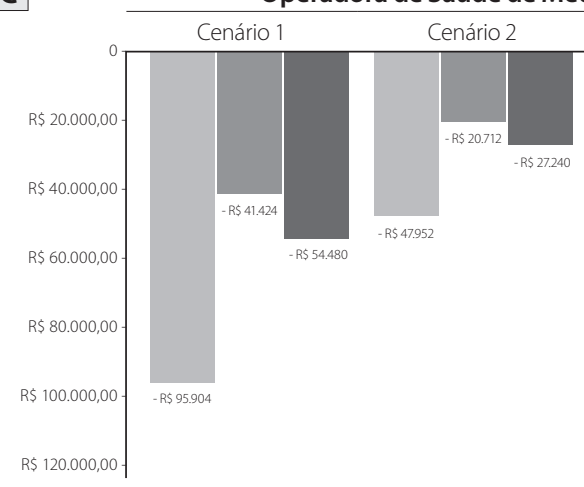

B

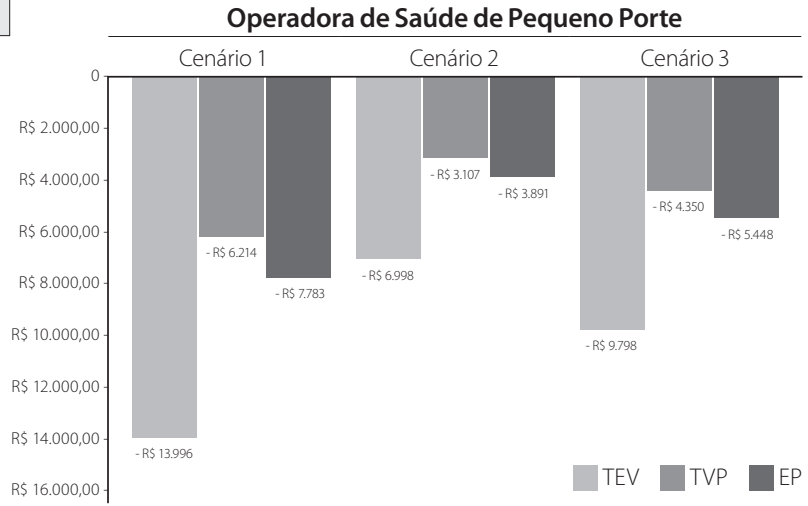

D

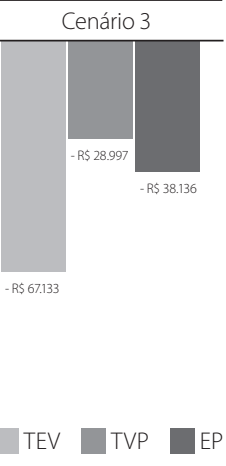

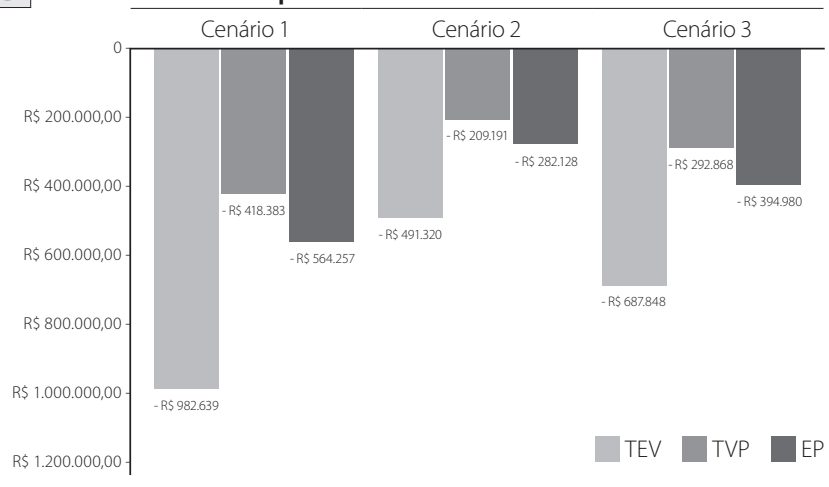

Figura 5. Resultados da análise de impacto orçamentário.

Tabela 8. Número médio de beneficiários por porte de operadora de saúde

\begin{tabular}{lll}
\hline Operadora de saúde & $\mathbf{2 0 1 6}$ & $\mathbf{2 0 1 7}$ \\
\hline Pequeno porte $(<20$ mil vidas) & 6.511 & 6.393 \\
\hline Médio porte (20-100 mil vidas) & 41.458 & 40.712 \\
\hline Grande porte ( $>100$ mil vidas) & 428.013 & 420.309 \\
\hline
\end{tabular}

em detalhes apenas para o mais conservador, cenário 2, na Tabela 9.

Para o Sistema de Saúde Suplementar completo, os resultados de economia de recursos variaram com todas as simulações entre aproximadamente $\mathrm{R} \$ 78$ milhões e $\mathrm{R} \$ 142$ milhões no cenário 1 e entre R\$ 55 milhões e R\$ 99 milhões no cenário 3. Em operadoras de saúde de grande porte, essa variação apresentou-se entre R\$ 699 mil e R\$1,2 milhões no cenário 1 e entre R\$ 489 mil e R\$ 885 mil no cenário 3. Nas operadoras de médio porte, a economia variou entre $\mathrm{R} \$ 67$ mil e R\$ 122 mil no cenário 1 e entre R\$ 47 mil e R\$ 85 mil no cenário 3. Finalmente, para operadoras de pequeno porte, os resultados variaram entre $\mathrm{R} \$ 10$ mil e $\mathrm{R} \$ 19$ mil no cenário 1 e entre R\$ 7 mil e R\$13 mil no cenário 3.

Assim como na análise de custo-minimização, o parâmetro mais sensível da análise foi o tempo de hospitalização de ambos os comparadores em todas as perspectivas e cenários (Figura 6).

\section{Discussão}

Os resultados da análise de custo-minimização e impacto orçamentário demonstraram que a rivaroxabana é um anticoagulante oral com potencial para reduzir custos de tratamento de TEV no Sistema de Saúde Suplementar do Brasil.

O tratamento com rivaroxabana apresentou menor custo do que o tratamento com enoxaparina/varfarina nas análises realizadas neste estudo. Entre os custos analisados, o principal direcionador do resultado favorável à rivaroxabana foi a redução do tempo de internação hospitalar, uma vez que a rivaroxabana é administrada como monoterapia oral e não necessita de monitoramento e ajustes de dose frequentes de acordo com parâmetros da coagulação. Essa redução do tempo de internação hospitalar dos pacientes tratados com rivaroxabana foi confirmada pelo estudo observacional XALIA, que recrutou mais de 5 mil pacientes em 21 países e comparou os resultados de efetividade e segurança dos tratamentos com rivaroxabana e terapia anticoagulante padrão (tratamento inicial com HNF, HBPM ou fondaparinux sobreposto e seguido de AVK) para TVP por pelo menos três meses. Nessa coorte, a média de duração de internação hos- 
Tabela 9. Resultados das análises de sensibilidade - impacto orçamentário - cenário 2 (market share de 50\% para rivaroxabana)

\begin{tabular}{|c|c|c|c|c|}
\hline Parâmetro & Variação & Mínimo & Custo-base & Máximo \\
\hline \multicolumn{5}{|l|}{ Sistema de Saúde Suplementar inteiro } \\
\hline Dias de hospitalização (E/V) & $\pm 20 \%$ & $-R \$ 39.441 .897,29$ & -R\$ 55.417.163,86 & -R\$ 71.392.430,43 \\
\hline Dias de hospitalização (rivaroxabana) & $\pm 20 \%$ & $-R \$ 70.212 .507,93$ & $-R \$ 55.417 .163,86$ & $-R \$ 40.621 .819,79$ \\
\hline Custo enoxaparina & $\pm 10 \%$ & $-R \$ 52.704 .728,12$ & -R\$ 55.417.163,86 & $-R \$ 57.540 .106,08$ \\
\hline Incidência EP & $0,65-0,73$ & $-R \$ 53.571 .617,18$ & $-R \$ 55.417 .163,86$ & $-R \$ 57.263 .197,55$ \\
\hline Incidência TVP & $0,45-0,51$ & $-R \$ 53.944 .032,98$ & -R\$ 55.417.163,86 & $-R \$ 56.891 .285,75$ \\
\hline Taxa de crescimento beneficiários & $(-0,7 \%)-(-2,7 \%)$ & $-R \$ 54.909 .999,49$ & $-R \$ 55.417 .163,86$ & $-R \$ 56.038 .673,68$ \\
\hline Custo rivaroxabana & $\pm 7 \%$ & $-R \$ 55.530 .893,55$ & $-R \$ 55.417 .163,86$ & $-R \$ 55.304 .466,51$ \\
\hline \multicolumn{5}{|l|}{ Operadora de grande porte } \\
\hline Dias de hospitalização (E/V) & $\pm 20 \%$ & $-R \$ 349.764,22$ & $-R \$ 491.319,68$ & $-R \$ 632.875,15$ \\
\hline Dias de hospitalização (rivaroxabana) & $\pm 20 \%$ & $-R \$ 622.462,93$ & $-R \$ 491.319,68$ & $-R \$ 360.176,43$ \\
\hline Custo enoxaparina & $\pm 10 \%$ & $-R \$ 467.275,15$ & $-R \$ 491.319,68$ & $-R \$ 510.138,62$ \\
\hline Incidência EP & $0,65-0,73$ & $-R \$ 507.858,24$ & $-R \$ 491.319,68$ & $-R \$ 474.781,13$ \\
\hline Incidência TVP & $0,45-0,51$ & $-R \$ 477.856,87$ & $-R \$ 491.319,68$ & $-R \$ 503.746,90$ \\
\hline Taxa de crescimento beneficiários & $(-0,7 \%)-(-2,7 \%)$ & $-R \$ 494.395,43$ & $-R \$ 491.319,68$ & $-R \$ 487.208,34$ \\
\hline Custo rivaroxabana & $\pm 7 \%$ & $-R \$ 492.327,76$ & $-R \$ 491.319,68$ & $-R \$ 490.320,76$ \\
\hline \multicolumn{5}{|l|}{ Operadora de médio porte } \\
\hline Dias de hospitalização (E/V) & $\pm 20 \%$ & $-R \$ 34.284,57$ & $-R \$ 47.951,99$ & $-R \$ 61.619,42$ \\
\hline Dias de hospitalização (rivaroxabana) & $\pm 20 \%$ & $-R \$ 60.695,06$ & $-R \$ 47.951,99$ & $-R \$ 35.208,92$ \\
\hline Taxa de crescimento beneficiários & $(-0,7 \%)-(-2,7 \%)$ & $-R \$ 43.935,08$ & $-R \$ 47.951,99$ & $-R \$ 50.933,31$ \\
\hline Custo enoxaparina & $\pm 10 \%$ & $-R \$ 45.611,84$ & $-R \$ 47.951,99$ & $-R \$ 49.783,56$ \\
\hline Incidência EP & $0,65-0,74$ & $-R \$ 46.006,28$ & $-R \$ 47.951,99$ & $-R \$ 49.897,70$ \\
\hline Incidência TVP & $0,45-0,51$ & $-R \$ 45.880,79$ & $-R \$ 47.951,99$ & $-R \$ 48.987,59$ \\
\hline Custo rivaroxabana & $\pm 7 \%$ & $-\mathrm{R} \$ 48.049,95$ & $-R \$ 47.951,99$ & $-R \$ 47.854,93$ \\
\hline \multicolumn{5}{|l|}{ Operadora de pequeno porte } \\
\hline Dias de hospitalização (E/V) & $\pm 20 \%$ & $-R \$ 5.045,74$ & $-R \$ 6.998,23$ & $-R \$ 8.950,72$ \\
\hline Dias de hospitalização (rivaroxabana) & $\pm 20 \%$ & $-R \$ 8.841,96$ & $-R \$ 6.998,23$ & $-R \$ 5.154,50$ \\
\hline Taxa de crescimento beneficiários & $(-0,7 \%)-(-2,7 \%)$ & $-R \$ 7.089,24$ & $-R \$ 6.998,23$ & $-\mathrm{R} \$ 7.989,91$ \\
\hline Custo enoxaparina & $\pm 10 \%$ & $-R \$ 6.658,57$ & $-R \$ 6.998,23$ & $-R \$ 7.264,07$ \\
\hline Incidência EP & $0,65-0,74$ & $-\mathrm{R} \$ 7.192,80$ & $-R \$ 6.998,23$ & $-R \$ 7.679,23$ \\
\hline Incidência TVP & $0,45-0,51$ & $-R \$ 6.894,67$ & $-R \$ 6.998,23$ & $-R \$ 7.308,91$ \\
\hline Custo rivaroxabana & $\pm 7 \%$ & $-R \$ 7.012,40$ & $-R \$ 6.998,23$ & $-R \$ 6.984,18$ \\
\hline
\end{tabular}

TVP: trombose venosa profunda; EP: embolia pulmonar; R: rivaroxabana; E/V: enoxaparina mais varfarina.

pitalar foi de cinco dias no grupo da rivaroxabana e de 7,7 dias no grupo da anticoagulação-padrão (Ageno et al., 2016). A duração da internação hospitalar é a principal responsável pelo aumento nos custos hospitalares; dado que pacientes com TEV apresentam longo tempo de internação, a adoção de um regime que reduza esse tempo é estratégica para a redução de custos com o tratamento (Fernandez et al., 2015).

A AlO demonstrou que a introdução de rivaroxabana para o tratamento de TEV no Sistema de Saúde Suplementar brasileiro, em operadoras de saúde de pequeno, médio ou grande porte, com market share de $100 \%, 70 \%$ ou $50 \%$, apresenta potencial de redução de custos de 20\% a 42\%, comparada com enoxaparina/varfarina. Uma vez que a incidência de TEV é alta e a população elegível para o tratamento no Sistema de Saúde Suplementar Brasileiro é grande, a opção por uma terapia com potencial de economia de recursos pode beneficiar a gestão orçamentária do sistema de saúde.

Deve-se considerar a escassez de dados epidemiológicos nacionais de TEV para a perspectiva do Sistema de Saúde Suplementar uma limitação, dado que a população elegível 
Sistema todo - Cenário 2

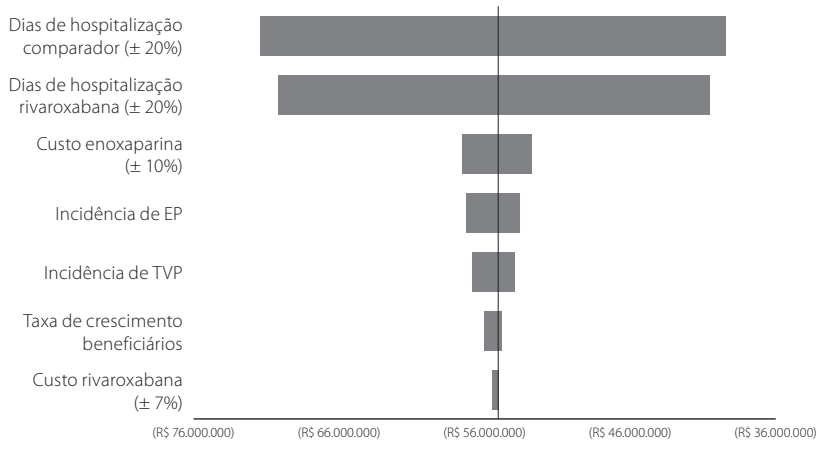

Médio Porte - Cenário 2

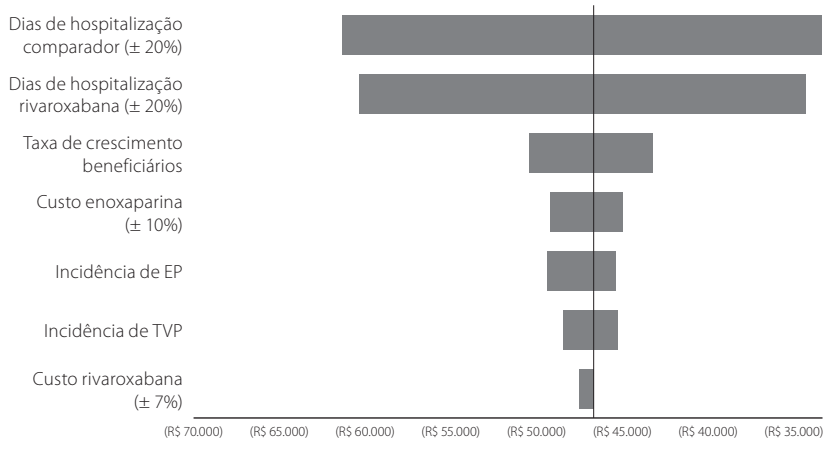

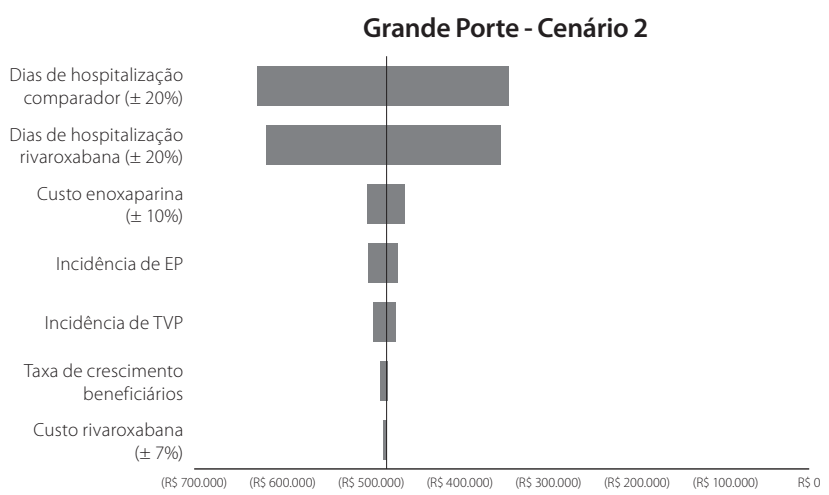

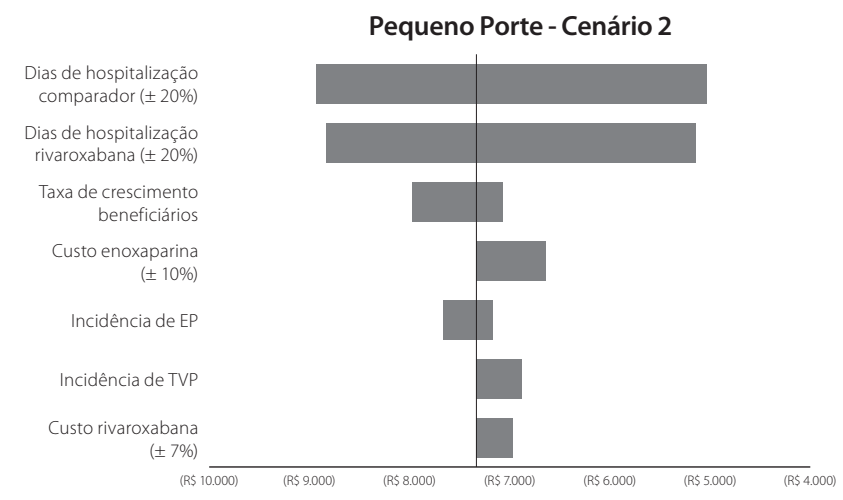

Figura 6. Diagramas de Tornado das Análises de Sensibilidade - Impacto Orçamentário - Cenário 2 (market share de 50\% para rivaroxabana).

para o tratamento foi estimada com base em estudos internacionais. Além disso, o número de beneficiários das operadoras de saúde em 2017 foi estimado com base em dados de 2016. Tendo em vista que desde 2015 essa taxa de crescimento de beneficiários passou a ser negativa, deve ser considerada a incerteza do parâmetro do número de beneficiários em 2017.

Assim como a maioria das avaliações farmacoeconômicas, este estudo de custo-minimização agrega diferentes fontes de dados, o que confere diferentes níveis de incerteza ao modelo. Os estudos EINSTEIN foram selecionados para embasar o racional clínico da análise, por serem os estudos pivotais de tratamento de TEV com rivaroxabana comparada ao tratamento-padrão. Essa seleção pode ser considerada uma limitação, uma vez que não foi realizada uma revisão sistemática da literatura a fim de detectar outras fontes de dados que pudessem contribuir para esse desenvolvimento. O parâmetro tempo de hospitalização foi o que apresentou maior sensibilidade, por ser o principal direcionador da diferença de custos entre as duas alternativas analisadas; entretanto, essa redução do tempo de internação hospitalar também foi notada com significância estatística nas análises dos estudos EINSTEIN e confirmada por um estudo observacional multicêntrico e internacional XALIA (Ageno et al., 2016; van Bellen et al., 2014). Outra limitação do modelo foi considerar que todos os pacientes aderiram ao tratamento e ao monitoramento: neste estudo não foram consideradas taxas de descontinuação ou mudança de tratamentos ao longo do tempo, eventos que podem acontecer na prática clínica de rotina. Apesar da incerteza dos parâmetros, todos os resultados demonstraram economia de recursos após a análise de sensibilidade, o que indica a robustez do modelo e confere maior credibilidade à avaliação realizada.

\section{Conclusão}

A rivaroxabana é um anticoagulante oral utilizado para o tratamento de TEV, com potencial de gerar economia de recursos, comparado ao tratamento com enoxaparina/varfarina no Sistema de Saúde Suplementar do Brasil, sendo o principal motivo dessa economia a redução do tempo de internação hospitalar dos pacientes.

\section{Referências bibliográficas}

Ageno W, Mantovani LG, Haas S, Kreutz R, Monje D, Schneider J, et al. Safety and effectiveness of oral rivaroxaban versus standard anticoagulation for the treatment of symptomatic deep-vein thrombosis (XALIA): an international, prospective, non-interventional study. Lancet Haematol. 2016;3(1):e12-21.

Agnelli G, Buller HR, Cohen A, Curto M, Gallus AS, Johnson M, et al.; AMPLIFY Investigators. Oral apixaban for the treatment of acute venous thromboembolism. N Engl J Med. 2013a;369(9):799-808. 
Agnelli G, Buller HR, Cohen A, Curto M, Gallus AS, Johnson M, et al.; PLIFY-EXT Investigators. Apixaban for extended treatment of venous thromboembolism. N Engl J Med. 2013b;368(8):699-708.

Bauersachs R, Berkowitz SD, Brenner B, Buller HR, Decousus H, Gallus AS, et al.; EINSTEIN Investigators. Oral rivaroxaban for symptomatic venous thromboembolism. N Engl J Med. 2010;363(26):2499-510.

Becattini C, Agnelli G. Treatment of venous thromboembolism with new anticoagulant agents. J Am Coll Cardiol. 2016;67(16):1941-55.

Brasil. Agência Nacional de Saúde Suplementar (ANS). Dados e Indicadores do Setor. Dados do Caderno de Informações da Saúde Suplementar. 2016 [cited 2015 05/05]; Available from: <http://www.ans.gov.br/perfildo-setor/dados-e-indicadores-do-setor $>$.

Brasil. Ministério da Saúde. Secretaria de Ciência, Tecnologia e Insumos Estratégicos. Departamento de Ciência e Tecnologia. Diretrizes metodológicas: Diretriz de Avaliação Econômica. 2a ed. Brasília: Ministério da Saúde; 2014.

Büller HR, Prins MH, Lensin AW, Decousus H, Jacobson BF, Minar E, et al.; EINSTEIN-PE Investigators. Oral rivaroxaban for the treatment of symptomatic pulmonary embolism. N Engl J Med. 2012;366(14):1287-97.

CBHPM - Classificação Brasileira Hierarquizada de Procedimentos Médicos. 2012.

Cheung YW, Middeldorp S, Prins MH, Pap AF, Lensing AW, Ten Cate-Hoek AJ, et al.; Einstein PTS Investigators Group. Post-thrombotic syndrome in patients treated with rivaroxaban or enoxaparin/vitamin K antagonists for acute deep-vein thrombosis. A post-hoc analysis. Thromb Haemost. 2016;116(4):733-8.

Câmara de Regulação do Mercado de Medicamentos - CMED. Lista de preços e medicamentos: preço fábrica e preço máximo de venda ao governo. Agência Nacional de Vigilância Sanitária. Secretaria Executiva. 2016; Atualização: dezembro de 2016.

Fernandez MM, Hogue S, Preblick R, Kwong WJ. Review of the cost of venous thromboembolism. Clinicoecon Outcomes Res. 2015;7:451-62.

Guyatt GH, Akl EA, Crowther M, Gutterman DD, Schuünemann HJ; American College of Chest Physicians Antithrombotic Therapy and Prevention of Thrombosis Panel. Executive summary: Antithrombotic Therapy and Prevention of Thrombosis, 9th ed: American College of Chest Physicians Evidence-Based Clinical Practice Guidelines. Chest. 2012;141(2 Suppl):7S-47S.

Heit JA. Epidemiology of venous thromboembolism. Nat Rev Cardiol. 2015;12(8):464-74.

Hirsh J, Dalen J, Anderson DR, Poller L, Bussey H, Ansell J, et al. Oral anticoagulants: mechanism of action, clinical effectiveness, and optimal therapeutic range. Chest. 2001a;119(1 Suppl):8S-21S.

Hirsh J, Warkentin TE, Shaughnessy SG, Anand SS, Halperin JL, Raschke R, et al. Heparin and low-molecular-weight heparin: mechanisms of action, pharmacokinetics, dosing, monitoring, efficacy, and safety. Chest. 2001b;119(1 Suppl):64S-94S.
Kearon C, Akl EA, Ornelas J, Blaivas A, Jimenez D, Bounameaux H, et al. Antithrombotic Therapy for VTE Disease: CHEST Guideline and Expert Panel Report. Chest. 2016;149(2):315-52.

McRae SJ, Ginsberg JS. Initial treatment of venous thromboembolism. Circulation. 2004;110(9 Suppl 1):13-9.

Ministério da Saúde. Secretaria de Ciência, Tecnologia e Insumos Estratégicos. Diretrizes metodológicas: análise de impacto orçamentário: manual para o Sistema de Saúde do Brasil. Departameno de Ciência e Tecnologia 1 ed, 1 reimpr. 2014.

Monreal M, Mahé I, Bura-Riviere A, Prandoni P, Verhamme P, Brenner B, et al. Pulmonary embolism: epidemiology and registries. Press Med. 2015;44(12 Pt 2):e377-83.

Prandoni P, Lensing AW, Cogo A, Cuppini S, Villalta S, Carta M, et al. The longterm clinical course of acute deep venous thrombosis. Ann Intern Med. 1996;125(1):1-7.

Prins MH, Lensing AW, Bauersachs R, van Bellen B, Bounameaux H, Brighton TA, et al.; EINSTEIN Investigators. Oral rivaroxaban versus standard therapy for the treatment of symptomatic venous thromboembolism: a pooled analysis of the EINSTEIN-DVT and PE randomized studies. Thromb J. 2013;11(1):21.

Ramacciotti E, Gomes M, de Aguiar ET, Caiafa JS, de Moura LK, Araújo GR, et al.; CLE-PTS Investigators. A cost analysis of the treatment of patients with post-thrombotic syndrome in Brazil. Thromb Res. 2006;118(6):699-704.

Raskob GE, Angchaisuksiri P, Blanco AN, Buller H, Gallus A, Hunt BJ, et al.; ISTH Steering Committee for World Thrombosis Day. Thrombosis: a major contributor to global disease burden. Arterioscler Thromb Vasc Biol. 2014;34(11):2363-71.

Schulman S, Kakkar AK, Goldhaber SZ, Schellong S, Eriksson H, Mismetti P, et al.; RE-COVER II Trial Investigators. Treatment of acute venous thromboembolism with dabigatran or warfarin and pooled analysis. Circulation. 2014;129(7):764-72.

Schulman S, Kearon C, Kakkar AK, Mismetti P, Schellong S, Eriksson H, et al.; RE-COVER Study Group. Dabigatran versus warfarin in the treatment of acute venous thromboembolism. N Engl J Med. 2009;361(24):2342-52.

Schulman S, Kearon C, Kakkar AK, Schellong S, Eriksson H, Baanstra D, et al.; RE-MEDY Trial Investigators; RE-SONATE Trial Investigators. Extended use of dabigatran, warfarin, or placebo in venous thromboembolism. N Engl J Med. 2013;368(8):709-18.

Silverstein MD, Heit JA, Mohr DN, Petterson TM, O'Fallon WM, Melton LJ $3 \mathrm{rd}$. Trends in the incidence of deep vein thrombosis and pulmonary embolism: a 25-year population-based study. Arch Intern Med. 1998;158(6):585-93.

van Bellen B, Bamber L, Correa de Carvalho F, Prins M, Wang M, Lensing AW. Reduction in the length of stay with rivaroxaban as a single-drug regimen for the treatment of deep vein thrombosis and pulmonary embolism. Curr Med Res Opin. 2014;30(5):829-37. 